\title{
The Effect of Several Commonly Used Antipsychotic Drugs on the Renal Function of Patients with Mental Illness
}

\author{
Yubiao Sun ${ }^{1 *}$, Huan Zhao ${ }^{2 *}$, Xiaoyu Jiang ${ }^{3 \#, ~ Q i a n g c a i ~ M a i ~}{ }^{4 \#}$ \\ ${ }^{1}$ Inspection Department of the Second People's Hospital of Mengshan County, Mengshan, China; ${ }^{2}$ Inspection \\ Department of Chongzuo Maternal and Child Health Care Hospital, Chongzuo, China; ${ }^{3}$ Inspection Department of \\ Nanning Third People's Hospital, Nanning, China; ${ }^{4}$ The Second Department of Guigang Orthopaedic Hospital of \\ Integrated Traditional Chinese and Western Medicine, Guigang, China
}

Correspondence to: Xiaoyu Jiang, "38669739@qq.com; Qiangcai Mai, "18007856271@163.com Keywords: Antipsychotic Drugs, Psychosis, Renal Function Test, Urea Nitrogen, $\beta 2$ Microglobulin, Uric Acid Received: December 30, $2021 \quad$ Accepted: February 4, 2022 Published: February 7, 2022

Copyright $\odot 2022$ by author(s) and Scientific Research Publishing Inc.

This work is licensed under the Creative Commons Attribution International License (CC BY 4.0).

http://creativecommons.org/licenses/by/4.0/

\section{(c) (i) Open Access}

\section{ABSTRACT}

Objective: To understand the effects of several commonly used antipsychotics on the renal function of patients with mental illness. Method: Collected patients with mental illness who were hospitalized in our hospital from January 2020 to June 2021, and selected as the research subjects patients with psychiatric disorders who were treated with 2 kinds of commonly used antipsychotic drugs; and collected $3 \mathrm{ml}$ of venous blood before treatment and one month after treatment for renal function tests; observed the changes of renal function indexes before and after treatment. Results: In the collected 694 patients with mental illness, before using antipsychotic drugs, the renal function indexes were BUN: $4.42 \pm 1.92 \mathrm{mmol} / \mathrm{l}$; Cr: $70.97 \pm 16.92 \mu \mathrm{mol} / \mathrm{l}$; CCr: $88.37 \pm 21.07 \mathrm{ml} / \mathrm{min} ; \beta 2-\mathrm{MG}: 1.67 \pm 0.61 \mathrm{mg} / \mathrm{L}$; UA: 359.90 $\pm 112.82 \mu \mathrm{mol} / \mathrm{l}$; CYS-C: $0.92 \pm 0.24 \mathrm{mg} / \mathrm{L}$. One month after using antipsychotics, BUN: 3.77 $\pm 1.37 \mathrm{mmol} / \mathrm{l}$; Cr: $70.46 \pm 16.71 \mu \mathrm{mol} / \mathrm{l}$; CCr: $87.78 \pm 20.63 \mathrm{ml} / \mathrm{min} ; \beta 2-\mathrm{MG}: 1.75 \pm 0.64$ $\mathrm{mg} / \mathrm{L}$; UA: $332.53 \pm 91.48 \mathrm{umol} / \mathrm{l}$; CYS-C: $0.92 \pm 0.24 \mathrm{mg} / \mathrm{L}$; the renal function indexes of urea nitrogen, $\beta 2$ microglobulin, uric acid and other items all changed significantly. The differences before and after treatment were statistically significant, $\mathrm{P}<0.01$. Conclusion: Several commonly used antipsychotic drugs have a greater impact on the renal function of patients with mental illness. During the treatment, the changes in renal function should be monitored regularly, if severe renal damage is found, the treatment plan or dosage should be adjusted in time to avoid endangering life.

${ }^{\star}$ First author.

"Corresponding author. 


\section{RESEARCH BACKGROUND}

Commonly used antipsychotic drugs mainly include clozapine, aripiprazole, risperidone, perphenazine, chlorpromazine, sulpiride, etc. These drugs have certain effects on the treatment of patients with mental illness [1-3]. Clozapine is a second-generation antipsychotic drug used earlier in psychiatry. It is an antagonist containing multiple receptors. It mainly antagonizes the central nervous serotonin 2 receptor to produce a strong antipsychotic effect. Clozapine is a drug that can control patients' symptoms such as excitement and impulse, and has outstanding effects on various hallucinations and delusions. Aripiprazole is also a second-generation antipsychotic. Risperidone is a drug mainly used to treat patients with acute or chronic schizophrenia, especially for the presence or absence of obvious symptoms and complicated affective mental illnesses such as anxiety and depression; it can also reduce the emotional symptoms associated with schizophrenia; risperidone also has a good effect on patients in the acute phase, and can still play a good clinical effect during the later maintenance treatment. Perphenazine is mainly used for the treatment of symptomatic mental illness, reactive psychosis and paranoid psychosis; it also has certain effects on simple and chronic schizophrenia; it also has a certain therapeutic effect on nausea, vomiting, hiccups, etc.; those with symptoms of anxiety or nervousness can use this drug in combination with other drugs for treatment. Chlorpromazine is a drug used to treat schizophrenia or other psychotic symptoms such as hallucinations, excitement, restlessness, delusions, etc.; it is not effective in the treatment of depression or numbness, and may even worsen the condition in patients with type II schizophrenia. Sulpiride is a drug that has a good effect on withdrawal, depression, apathy, hallucinations, stupor, and delusions; it has a good effect on various types of symptoms of schizophrenia; it also has a certain therapeutic effect on depression [4-6]. Although these drugs have different effects on mental illness, they also have certain side effects, especially kidney damage. In order to explore the renal damage caused by several commonly used antipsychotic drugs, the subjects were followed up and observed in this study, and the results are reported as follows.

\section{DATA AND METHODS}

\subsection{Research Objects}

694 subjects were all from psychiatric patients hospitalized in our hospital from January 2020 to June 2021, including 409 males, 285 females, male and female comparison, $\chi^{2}=44.3112, \mathrm{P}=0.0000$. Inclusion criteria: 1) Included in the cohort study according to the Chinese 3rd edition of the classification methods and diagnostic criteria of mental illness and the related schizophrenia diagnostic criteria of the 10th edition of the International Classification of Diseases; 2) before and after enrollment, no other drugs causing kidney damage were taken at the same time. Exclusion criteria: patients with related urinary or kidney disease. 3) All patients included in the study were selected for combined treatment of 2 antipsychotics among several commonly used antipsychotics according to the needs of the disease.

\subsection{Methods}

Took $3 \mathrm{ml}$ of fasting venous blood from patients before admission and one month after hospitalization; separated the serum and stored it in an ultra-low temperature refrigerator at $-80^{\circ} \mathrm{C}$. After the specimens were collected, performed a unified renal function test. The renal function indicators include urea nitrogen, Creatinine, endogenous creatinine clearance rate, $\beta 2$ microglobulin, uric acid, cystatin and other items.

\subsection{Instruments and Reagents}

Hitachi Automatic biochemical analyzer 7180 is used for instruments; the renal function index adopts the products of Beijing Jiuqiang Biotechnology Co., LTD., and the indoor quality control adopts Landau's quality control. All the reagents are used within the validity period, and all the renal function indexes have passed the ventricular interstitial evaluation of Guangxi Zhuang Autonomous Region Clinical Examina- 
tion center, and every result has reached excellent.

\subsection{Statistical Analysis}

Statistical software SSPS 24.0 was used for statistical analysis. The statistical data were expressed by $(\bar{X} \pm s)$, the chi-square test was used to compare the incidence of male and female psychiatric patients, and the $\mathrm{T}$ test was used to compare the mean data between normal distribution groups. $\mathrm{P}<0.05$ was considered as statistically significant difference.

\section{RESULTS}

In the collected 694 patients with mental illness, Comparing renal function indexes before treatment and one month after treatment, the differences in BUN, $\beta 2-M G$, UA and other items were statistically significant, The t values were 7.2598, 2.3837, and 4.9641, respectively, with $\mathrm{P}<0.01$. There was no significant difference in Cr, CCr, and CYS-C, and the t values were 0.5650, 0.5271, and 0.0000, respectively, with all $\mathrm{P}>0.05$. For details, see Table 1 .

\section{DISCUSSION}

Antipsychotic drugs have different levels of adverse reactions [7-13]. Clozapine has a strong sedative and anticholinergic effect, so there are many adverse reactions. Common adverse reactions include nausea, vomiting, and dizziness, drowsiness, weakness, dry mouth, hyperhidrosis, increased appetite, weight gain, salivation, constipation, tachycardia, abnormal electrocardiogram, orthostatic hypotension and other changes which can cause seizures or changes in electroencephalogram and can cause serious illness agranulocytosis; secondary infections and increased blood sugar can also cause central nervous system disorders or urinary incontinence. Clinical medications are used with caution or not. The adverse reactions of aripiprazole are relatively mild, with fewer symptoms such as weight gain or extrapyramidal reactions, and the patients have better tolerance. Adverse reactions mainly include urinary incontinence, drowsiness, headache, anxiety, insomnia, and inability to sit still. The main common adverse reactions of risperidone are: dizziness, headache, dry mouth, anxiety, and insomnia. The relatively rare adverse reactions include: nausea, vomiting, fatigue, drowsiness, decreased concentration, abdominal pain, constipation, indigestion, priapism, apathy, weakness of ejaculation, difficulty erection, blurred vision, urinary incontinence, skin rash, rhinitis And other allergic reactions. It can also cause extrapyramidal reactions, such as stiffness, muscle tension, acute dystonia, bradykinesia, salivation, tremor, akathisia, etc. This symptom can be eliminated by reducing the dose or giving anti-Parkinson's syndrome drugs. Occasionally, symptoms of reflex tachycardia, high blood pressure, or orthostatic hypotension may occur. There will be edema, increased

Table 1. Comparison of the effects of combined treatment of two kinds of commonly used antipsychotic drugs on various indicators of renal function.

\begin{tabular}{ccccccc}
\hline Group & $\begin{array}{c}\text { Urea } \\
\text { nitrogen }\end{array}$ & Creatinine & $\begin{array}{c}\text { Endogenous } \\
\text { creatinine } \\
\text { clearance }\end{array}$ & $\begin{array}{c}\beta 2 \\
\text { globulin }\end{array}$ & Uric acid & $\begin{array}{c}\text { Cystatin } \\
\text { C }\end{array}$ \\
\hline $\begin{array}{c}\text { Prior treatment } \\
\text { One month } \\
\text { after treatment }\end{array}$ & $4.42 \pm 1.92$ & $70.97 \pm 16.92$ & $88.37 \pm 21.07$ & $1.67 \pm 0.61$ & $359.90 \pm 112.82$ & $0.92 \pm 0.24$ \\
t value & 7.2598 & 0.5650 & 0.5271 & 2.3837 & 4.9641 & 0.0000 \\
P value & 0.0000 & 0.2861 & 0.2991 & 0.0086 & 0.0000 & 0.5000 \\
\hline
\end{tabular}


liver enzyme levels, and weight gain. Occasionally, symptoms of water intoxication may occur due to the patient's irritability and thirst or imbalance of antidiuretic hormone secretion (SIADH). It can cause an increase in blood prolactin concentration, and the main symptoms are: menstrual disorders, amenorrhea, gynecomastia, galactorrhea, etc. Occasionally, seizures, temperature disorders, malignant symptom clusters, and tardive dyskinesias are seen. There are also reports of mild neutrophil and platelet count declines. The main adverse reactions of perphenazine are: extrapyramidal symptoms, such as acute dystonia, stiffness, tremor, bradykinesia, salivation, akathisia, etc. Long-term use of large amounts of drugs can cause tardive motor dysfunction. It can cause an increase in the concentration of prolactin in the blood. The main symptoms are: menstrual disorders, amenorrhea, male feminized breasts, and galactorrhea. Dizziness, sweating, dry mouth, fatigue, blurred vision, tachycardia, constipation, etc. may occur. Uncommon adverse reactions mainly include neutropenia, toxic liver damage and orthostatic hypotension. Occasionally, symptoms such as malignant syndrome and allergic rash are seen. The main adverse reactions of chlorpromazine are dry mouth, fatigue, palpitations, lethargy, constipation and leukopenia, and occasionally breast enlargement, lactation, amenorrhea, obesity, tachycardia, arrhythmia, postural hypotension, atrioventricular block and even death. Extrapyramidal reactions include dyskinesia, tremor and so on. It can cause retinopathy, cornea and lens opacity, increased intraocular pressure, jaundice, liver damage. Some allergic reactions can occur, such as asthma, allergic purpura, contact dermatitis, skin rash, extraneous dermatitis, etc. The main adverse reactions of sulpiride are chest tightness, transient ecg changes, pulse frequency, increased or decreased blood pressure, etc. It can be seen that sometimes there are mild extrapyramidal symptoms, which can cause weight gain, inability to ejaculate, lactation, abnormal menstruation, restlessness, excitement, insomnia, gastrointestinal reaction, thirst, sleepiness, headache, fever, sweating, dyskinesia, dysuria, etc., and also rash, itching and other allergic reactions. All kinds of drugs have certain side effects, so they have certain influence on liver and kidney functions [14, 15].

The results of this study showed that after the use of antipsychotic drugs for one month, the renal function indexes of patients with mental illness were compared, the differences of BUN, $\beta 2-\mathrm{MG}$, UA and other items were statistically significant, $\mathrm{P}<0.01$; There was no significant difference in $\mathrm{Cr}, \mathrm{CCr}, \mathrm{CYS}-\mathrm{C}$ and other items $(\mathrm{P}>0.05)$, indicating that taking antipsychotic drugs had a certain effect on renal function. According to the statistical results, the drugs used in our hospital accounted for $38 \%$ of single psychotropic drugs, $44 \%$ of 2 drug combinations, $15 \%$ of 3 drug combinations and $4 \%$ of 4 drug combinations. The study found that the combination of multiple drugs had a greater effect on renal function than did monotherapy.

\section{CONCLUSION}

To sum up, several common antipsychotic drugs have a great impact on renal function in patients with mental diseases. During the treatment period, the changes of renal function should be monitored regularly, if severe renal damage is found, the treatment plan or dosage should be adjusted in time to avoid endangering life. When the use of unilateral drugs is effective, multiple combinations should be avoided to reduce side effects.

\section{ACKNOWLEDGEMENTS}

This project has been approved, with strong support from the functional departments of the hospital and the help of my colleagues. I will express my heartfelt thanks for their support and help here! I wish them good health, smooth work and all the best!

\section{LIMITATIONS OF THE RESEARCH}

Because the study subjects were all from the patients hospitalized in the hospital, there are some differences, and could not fully represent the situation of all the patients in the other areas, so there are certain limitations. 


\section{FUND PROJECT}

Research on the clinical application value of Urine sample detection of CYS-C in a subtopic of National General Technology Research and Application of National Quality Foundation Research and Application of National R \& D Program (NO.: 2019YFF0216502).

\section{CONFLICTS OF INTEREST}

The authors declare no conflicts of interest regarding the publication of this paper.

\section{REFERENCES}

1. Xu, J.J., Hou, X.M., Bi, Z.K., et al. (2016) Comparative Study of the Effects of Clozapine, Chlorpromazine, and Risperidone on the Renal Function of Patients with Schizophrenia. Chinese Journal of Clinicians, 44, 67-68.

2. Zhou, Y., Fu, Q.W., Wu, C.D., et al. (2018) Effects of Low-Dose Metformin on Bone Metabolism, Insulin and Renal Function in Patients with Schizophrenia Caused by Oral Clozapine with Abnormal Glucose Metabolism. Hebei Medicine, 24, 111-115.

3. Wang, G.Q. and Yi, X.R. (2017) Changes in Related Metabolic Indexes and Liver and Kidney Function during the Treatment of Schizophrenia with Risperidone. Laboratory Medicine and Clinics, 14, 1085-1086.

4. Yuan, D.R., Zhang, X.R., Lu, R., et al. (2015) The Effect of Blood Lipid Levels on the Plasma Concentration of Olanzapine in Patients with Schizophrenia. Sichuan Mental Health, No. 4, 310-313.

5. Song, J.D. and Wang, W. (2017) The Effect of Astragalus Adjuvant Treatment of Chronic Schizophrenia with Metabolic Syndrome on the Antioxidant Capacity and Liver and Kidney Functions of Patients. Chinese Journal of Biochemical Pharmaceutics, No. 7, 157-158.

6. Sun, W.W., Cheng, Y.Q., Chen, X.Y., et al. (2017) Study on the Blood Concentration and Clinical Efficacy of Paliperidone in the Treatment of Schizophrenia. China Journal of Modern Medicine, 27, 126-130.

7. Ruan, S.F. and Bu, L.H. (2018) Clinical Pharmacist's Pharmaceutical Care for Patients with Schizophrenia and Diabetes Mellitus. Evaluation and Analysis of Medication in Chinese Hospitals, 18, 1140-1142.

8. Han, Y.C., Jia, J.M., Huang, T.X., et al. (2016) The Effect of Metformin on Glucose and Lipid Metabolism in Patients with Schizophrenia Complicated with Metabolic Syndrome and Its Safety Analysis. Journal of Psychiatry, 29, 443-447.

9. Liu, S.H. (2015) Analysis of the Efficacy of Amisulpride and Risperidone in the Treatment of Negative Symptoms of Schizophrenia. Jiangxi Medicine, 50, 1227-1229.

10. Zhang, M.Y. (2018) Schizophrenia Requires Long-Term Treatment. Chinese Journal of Psychiatry, 51, 65-66.

11. Zhang, Q., Liu, Q., Zhou, J., et al. (2017) Comparative Study of the Effects of Clozapine, Chlorpromazine and Risperidone on the Renal Function of Patients with Schizophrenia. World Clinical Drugs, 38, 822-825.

12. Zou, Y.Z. and Kong, Y.Y. (2019) Application and Safety Analysis of Combined Use of Antipsychotic Drugs in the Treatment of Patients with Schizophrenia. China Journal of Modern Medicine, 21, 101-103.

13. Wang, G.Y. (2018) Observation on the Effect of Antipsychotic Drugs on Myocardial Enzymes and Electrocardiogram in Patients with Schizophrenia. Electronic Journal of Clinical Medicine Literature, 5, 146.

14. Liu, C.Q. and Zhang, H. (2020) Comparative Observation of the Effects of Clozapine, Chlorpromazine, and Risperidone on the Renal Function of Patients with Schizophrenia. Chinese Medical Guide, 18, 63-64.

15. Liu, X.S., Li, J.Y. and Chen, F.Y. (2020) Comparative Analysis of the Effects of Clozapine, Chlorpromazine and Risperidone on the Renal Function of Patients with Schizophrenia. Gems of Health, 8, 46. 